\title{
Weyl doubling
}

\section{Rashid Alawadhi, David S. Berman and Bill Spence}

Centre for Research in String Theory, School of Physics and Astronomy, Queen Mary University of London, Mile End Road, London, E1 4NS, U.K.

E-mail: r.alawadhi@qmul.ac.uk, d.s.berman@qmul.ac.uk, w.j.spence@qmul.ac.uk

ABSTRACT: We study a host of spacetimes where the Weyl curvature may be expressed algebraically in terms of an Abelian field strength. These include Type D spacetimes in four and higher dimensions which obey a simple quadratic relation between the field strength and the Weyl tensor, following the Weyl spinor double copy relation. However, we diverge from the usual double copy paradigm by taking the gauge fields to be in the curved spacetime as opposed to an auxiliary flat space.

We show how for Gibbons-Hawking spacetimes with more than two centres a generalisation of the Weyl doubling formula is needed by including a derivative-dependent expression which is linear in the Abelian field strength. We also find a type of twisted doubling formula in a case of a manifold with Spin(7) holonomy in eight dimensions.

For Einstein Maxwell theories where there is an independent gauge field defined on spacetime, we investigate how the gauge fields determine the Weyl spacetime curvature via a doubling formula. We first show that this occurs for the Reissner-Nordström metric in any dimension, and that this generalises to the electrically-charged Born-Infeld solutions. Finally, we consider brane systems in supergravity, showing that a similar doubling formula applies. This Weyl formula is based on the field strength of the p-form potential that minimally couples to the brane and the brane world volume Killing vectors.

KEYwords: Classical Theories of Gravity, Gauge-gravity correspondence

ArXiv EPRINT: 2007.03264 


\section{Contents}

1 Introduction 1

2 Weyl doubling $\quad 3$

2.1 Spacetime classification 3

2.2 Invariants 4

2.2.1 Taub-NUT 5

2.2.2 Plebanski-Demianski 6

$\begin{array}{lll}2.2 .3 & \text { Eguchi-Hanson } & 7\end{array}$

2.2.4 Singly rotating Myers-Perry 8

3 The Gibbons-Hawking metrics $\quad 9$

4 An eight-dimensional example with $\operatorname{Spin}(7)$ holonomy 12

5 Reissner-Nordström and Born-Infeld $\quad 14$

$\begin{array}{lll}5.1 & \text { Reissner-Nordström } & 14\end{array}$

$\begin{array}{lll}5.2 & \text { Born-Infeld } & 15\end{array}$

6 Brane solutions $\quad 17$

$\begin{array}{ll}\text { 6.1 String in five dimensions } & 17\end{array}$

$\begin{array}{lll}6.2 \text { M2 brane } & 18\end{array}$

$\begin{array}{lll}6.3 & \text { D3 brane } & 19\end{array}$

$\begin{array}{lll}6.4 \text { M5 brane } & 19\end{array}$

$\begin{array}{llr}7 & \text { Discussion } & 20\end{array}$

A Myers-Perry pentad $\quad 22$

\section{Introduction}

The "double copy" and its inverse the "single copy" have received considerable attention in the past few years, as they provide an intriguing link between gauge theories and gravity. The double copy refers to moving from gauge theory to gravity while the single copy is the inverse map (there is also a "zeroth copy" to a scalar theory). This relationship, as a map between perturbative scattering amplitudes in gauge theory and gravity, was first studied in [1-3]. A tree-level proof has been given [3-11], where it has a stringy origin [12], and support for its existence at loop level found in [2, 13-43].

Subsequently the double/single copy was applied to some exact classical solutions. The Schwarzschild solution was shown to single copy to an electric charge [44], the Taub-NUT 
solution to a magnetic monopole [45] and the Eguchi-Hanson solution mapped to a selfdual gauge field [46], for example. More general topologically non-trivial solutions have been double copied in the work of [47]. Other work examining symmetries of the linearised double copy is [48-56]. More recent work has developed a wide variety of analysis applied to the double copy [57]-[93].

In [60] four-dimensional type D spacetimes were investigated, using a double copy formula for the Weyl curvature spinor in terms of a Maxwell spinor. Duality symmetries of gauge theories and their relationship to solution-generating maps in gravity have also been studied recently from the point of view of the double copy [75, 77]. The earlier work of [94] had used a self-dual Maxwell field, defined in terms of a Killing vector on the spacetime, in order to study how the Weyl tensor transformed under $\operatorname{sl}(2, R)$, and noted in particular that if the Weyl tensor was given by a suitable function quadratic in the Maxwell field, then the $s l(2, R)$-transformed metric also had a Weyl tensor satisfying this property. In [77] we, with Peinador Veiga, studied various metrics for which this is the case, showing how they transform under duality. This work, and that of [60], suggested that it would be interest to study cases where the Weyl tensor is given in terms of an Abelian gauge field, by what we will call "Weyl doubling".

In this paper we would thus like to explore classes of gravity and gravity-gauge field systems where the Weyl curvature of a spacetime is given as a quadratic function of an Abelian field strength which is also defined on the spacetime. Crucially we differ from the normal double copy scheme as our gauge field will be taken to be in the curved space time rather than some auxiliary flat background. We will investigate two such classes - the first where the gauge field is defined using intrinsic geometric properties of the spacetime, and the second where it is an additional field in the theory.

This paper is organised as follows. In section 2 we introduce the definition of purely algebraic Weyl doubling. We then show that the spacetimes obeying Weyl doubling satisfy the conditions for type $\mathrm{D}$ in four and higher dimensions. The independent scalar curvature invariants are then functions of the invariants constructed from the field strength, and the Weyl-NP scalars are in turn functions of the Maxwell-NP scalars. We give a number of examples.

In section 3 we then study gravitational instantons where the curvature satisfies a self-duality condition. For the Gibbons-Hawking metrics, the anti-self-dual two form field strength derived from the Killing vector is expanded in the triplet of anti-self-dual two forms and we show that these coefficients determine the Weyl tensor of the metric via two terms - the first being a direct tensor doubling formula as in section 2, and the second involving derivatives of the Abelian field strength. Based on this we give a derivative correction to the Weyl doubling formula. In higher dimensions, we find a manifold with Spin(7) holonomy that obeys a doubling construction using a type of twisted Maxwell field.

We next consider examples where there is an independent gauge field defined on the spacetime and investigate if the gauge fields in these gravity-gauge systems obey a doubling formula for the Weyl tensor. In section 4 we first give an example for the case of the Reissner-Nordström metric in any dimension, showing that the Weyl tensor is given by a doubling formula based on the external electromagnetic field strength. This formula applies 
to both the extremal and the non-extremal Reissner-Nordström solutions. We show that this generalises to the charged Born-Infeld solution in any dimension. In section 5 we then consider brane systems in supergravity. Here we show that the components of the Weyl tensor are given by a simple doubling formula based on the field strength of the p-form that minimally couples to the brane world volume and the brane Killing vectors. We finish with a discussion of the results and future work in section 6 .

\section{Weyl doubling}

\subsection{Spacetime classification}

The central object of study will be the "doubling" formula, where a tensor, $C[F]$ with the algebraic symmetries of the Weyl tensor in $D$ dimensions can be constructed from an $n$-form $F=d A$ (with the gauge field $A$ an $n-1$ form) as follows:

$$
C_{\mu \nu \rho \sigma}[F]=F_{\mu \nu} \cdot F_{\rho \sigma}-F_{\rho \mu} \cdot F_{\nu \sigma}-\frac{6}{D-2} g_{\mu \rho} F_{\nu} \cdot F_{\sigma}+\left.\frac{3}{(D-1)(D-2)} g_{\mu \rho} g_{\nu \sigma} F \cdot F\right|_{s},
$$

where a dot product means to contract all non-visible indices between the two terms, for example for an $n$-form, $F_{\mu \nu} \cdot F_{\rho \sigma}=F_{\mu \nu \lambda_{3} \ldots \lambda_{n}} F_{\mu \nu} \lambda_{3} \ldots \lambda_{n}$. The symbol " $\left.\right|_{s}$ " above applies to the expression on the right-hand side of the equation, and it means to anti-symmetrise in the indices $\mu, \nu$ and in $\rho, \sigma$, with unit weight.

We wish to study cases where the spacetime Weyl tensor $C_{\mu \nu \rho \pi}[g]$ is proportional to this expression

$$
C_{\mu \nu \rho \pi}[g]=\frac{1}{\sigma} C_{\mu \nu \rho \pi}[F]
$$

for some function $\sigma$. In a later example we will exhibit a derivative corrected expression where the right-hand side of (2.2) contains further terms determined by derivatives of $F$. The field strength $F$ is closed and required to be divergence-free. (Note that the field is defined on the spacetime with metric $g_{\mu \nu}$ thus the divergence equation is non-trivial.)

Let us first consider the case $n=2$ where we have a normal Abelian two form field strength. (The cases with $n>2$ will be relevant when we discuss supergravity brane solutions.) One can identify two cases where the divergence equation follows automatically. The first is where

$$
F_{\mu \nu}=2 \partial_{[\mu} K_{\nu]}
$$

for a Killing vector $K_{\mu}$, and the second is where there is a self-duality condition

$$
F_{\mu \nu}=\phi_{\mu \nu}^{\rho \sigma} F_{\rho \sigma}
$$

for some covariantly constant four form $\phi$ on the manifold. (The Killing vector-defined field strength may also satisfy a self-duality condition of course, or have this imposed.) We will study examples of both situations below.

In the first case, where we construct $F$ from the Killing vector $K$, we note that $K^{\mu} F_{\mu \nu}$ is closed. This follows from application of Cartan's formula for the Lie derivative:

$$
\mathcal{L}_{K} F=i_{K} d F+d i_{K} F
$$


which means for closed $F$ and $K$ Killing then $d i_{K} F=0$. Locally we can solve this condition to write $K^{\mu} F_{\mu \nu}$ an exact form so that

$$
K^{\mu} F_{\mu \nu}=\partial_{\nu} \sigma
$$

A solution to this (up to the addition of an arbitrary constant) is

$$
\sigma=K^{\mu} K_{\mu}
$$

In four dimensions, an analysis of the action of duality transformations [77, 94] shows that this is the same $\sigma$ as in equation (2.2). See also [95, 96] where this was seen earlier using the spinor formalism in Type D spacetimes and more recently $[97,98]$ for related work in higher dimensions.

The formula (2.2) naturally implies some conditions on the spacetime. Write the eigenvector equation for $F$ as

$$
F_{\mu}{ }^{\nu} k_{\nu}=\lambda_{k} k_{\mu}
$$

for eigenvector $k_{\mu}$ and eigenvalue $\lambda_{k}$. Note that the eigenvectors are necessarily null. Since $F$ is antisymmetric, its eigenvalues form into pairs with opposite signs (and one zero eigenvalue if $D$ is odd). Then (2.2) implies that

$$
C_{\mu \nu \rho \sigma} k^{\nu} k^{\sigma}=\Lambda k_{\mu} k_{\rho}
$$

with

$$
\Lambda=\frac{3}{2(D-1)(D-2)}\left[(D-1)(D-4) \lambda_{k}^{2}+F_{\alpha \beta} F^{\beta \alpha}\right] .
$$

This implies that the eigenvector $k_{\mu}$ is a principal null direction of the Weyl tensor. Equation (2.2) also implies that

$$
k_{[\alpha} C_{\mu] \nu[\rho \sigma} k_{\pi]} k^{\nu}=0 .
$$

and similarly for a second eigenvector $l_{\mu}$ with a different eigenvalue. Thus generically there are two principal null directions satisfying (2.11) which implies that the spacetime satisfies the conditions for falling within the type D class in the appropriate higher-dimensional classification [99] (see also the overview [100]). A special case occurs when the eigenvectors are not independent and so there is only one principal null direction. This occurs when $F$ obeys a self-duality relation in which case the two eigenvectors are identical. The spacetime is then said to fall into the type II class.

\subsection{Invariants}

Equation (2.2) implies that scalar invariants constructed from the Weyl tensor (and its dual where there is a suitable four-form on the manifold) are functions of the traces

$$
\left(F^{n}\right)=F_{\mu_{2}}^{\mu_{1}} F_{\mu_{3}}^{\mu_{2}} \ldots F^{\mu_{n}}{ }_{\mu_{1}} .
$$

We will use a similar notation for traces of products of Weyl tensors (or their duals), for example

$$
\left(C^{3}\right)=C_{\rho \pi}^{\mu \nu} C_{\alpha \beta}^{\rho \pi} C_{\mu \nu}^{\alpha \beta}
$$


In the four-dimensional case, then,

$$
\begin{aligned}
\left(C^{2}\right) & =\frac{1}{\sigma^{2}}\left(\frac{9}{4}\left(F^{2}\right)^{2}-3\left(F^{4}\right)\right), \\
\left(C C^{*}\right) & =\frac{1}{\sigma^{2}}\left(\left(F^{2}\right)\left(F F^{*}\right)+2\left(F^{3} F^{*}\right)\right), \\
\left(C^{3}\right) & =\frac{1}{\sigma^{3}}\left(-\frac{61}{2}\left(F^{6}\right)+\frac{201}{8}\left(F^{2}\right)\left(F^{4}\right)-\frac{41}{8}\left(F^{2}\right)^{3}\right) \\
\left(C^{2} C^{*}\right) & =\frac{1}{\sigma^{3}}\left(\frac{21}{2}\left(F^{3}\left(F^{3}\right)^{*}\right)-\left(F^{2}\right)\left(F^{3} F^{*}\right)+\left(-\frac{5}{2}\left(F^{2}\right)^{2}+2\left(F^{4}\right)\right)\left(F F^{*}\right)+\frac{11}{2}\left(F^{5} F^{*}\right)\right),
\end{aligned}
$$

and so on. We define $F_{\mu \nu}^{*}=\frac{1}{2} \epsilon_{\mu \nu \rho \sigma} F^{\rho \sigma}$, and $\left(F^{3}\right)^{*}{ }_{\mu \nu}=\frac{1}{2} \epsilon_{\mu \nu \rho \sigma} F^{\rho \alpha} F_{\alpha \beta} F^{\beta \sigma}$ in the above. For four-dimensional vacuum spacetimes, the invariants (2.14) form an independent basis (under algebraic relationships) for the set of scalar invariants [101]. In addition, traces of higher powers of $F$ are related to those of lower powers by the recursion relation

$$
\left(F^{2 n}\right)=\frac{1}{2}\left(F^{2 n-2}\right)\left(F^{2}\right)-\frac{1}{8}\left(\left(F^{2}\right)^{2}-2\left(F^{4}\right)\right)\left(F^{2 n-4}\right) .
$$

Using this, one can express any $\left(F^{n}\right)$ in terms of $\left(F^{2}\right)$ and $\left(F^{4}\right)$, and hence all scalar curvature invariants in the vacuum case are functions of these two traces. There are analogous results in $D>4$.

If one block-diagonalises $F$ as

$$
F=\left(\begin{array}{cccc}
0 & x & 0 & 0 \\
-x & 0 & 0 & 0 \\
0 & 0 & 0 & y \\
0 & 0 & -y & 0
\end{array}\right)
$$

then $\left(F^{2}\right)=-2\left(x^{2}+y^{2}\right)$ and $\left(F^{4}\right)=2\left(x^{4}+y^{4}\right)$ and the invariants are functions of these combinations. If $x$ and $y$ are proportional then $\left(F^{2}\right)$ is the only independent function.

Similar arguments to those above apply to express the Weyl-NP scalars in terms of the independent Maxwell-NP scalars, depending on the dimension. We now give some examples to illustrate the above.

\subsubsection{Taub-NUT}

The Taub-NUT metric can be written in the form [102]

$$
d s^{2}=-f(r)(d t-2 n \cos \theta d \phi)^{2}+f(r)^{-1} d r^{2}+\left(r^{2}+n^{2}\right) d \Omega_{2}^{2}
$$

with

$$
f(r)=\frac{r^{2}-2 m r-n^{2}}{r^{2}+n^{2}} .
$$

From the Killing vector $K^{\mu}=(1,0,0,0)$ define the Maxwell field $F_{\mu \nu}=2 \partial_{[\mu} K_{\nu]}$. Then we have the Weyl doubling formula [77]

$$
C_{\mu \nu \rho \pi}^{+}=\frac{1}{\sigma^{+}} C_{\mu \nu \rho \pi}\left[F^{+}\right]
$$


with $\sigma^{+}=-\frac{m+i n}{r+i n}=:-\frac{m_{+}}{r_{+}}$. The eigenvalues of $F^{+}$are $\frac{m_{+}}{r_{+}^{2}}(1,1,-1,-1)$ with corresponding eigenvectors

$$
\begin{array}{ll}
m^{\mu}=\frac{1}{\sin \theta \sqrt{2\left(r^{2}+n^{2}\right.}}(-2 n \cos \theta, 0,-i, 0), & l^{\mu}=\frac{1}{\sqrt{\lambda}}(\lambda, 1,0,0), \\
\bar{m}^{\mu}=\frac{1}{\sin \theta \sqrt{2\left(r^{2}+n^{2}\right.}}(-2 n \cos \theta, 0, i, 0), & n^{\mu}=\frac{1}{\sqrt{\lambda}}(-\lambda, 1,0,0),
\end{array}
$$

with $\lambda=\left(r^{2}+n^{2}\right) /\left(n^{2}+2 m r-r^{2}\right)$. These form a null tetrad, with $l_{\mu} n^{\mu}=-1, m_{\mu} \bar{m}^{\mu}=1$ and the remaining inner products zero.

As $F^{+}$has repeated eigenvalues, the only independent trace is

$$
\left(F^{+} F^{+}\right)=-4 \frac{m_{+}^{2}}{r_{+}^{4}}
$$

leading to

$$
\left(C^{+} C^{+}\right)=24 \frac{m_{+}^{2}}{r_{+}^{6}} .
$$

All other scalar invariants are functions of this expression and its conjugate.

The formula (2.19) implies corresponding relationships amongst the NP scalars. In this case, for example, the Maxwell-NP scalars are $\phi_{0}=F_{\mu \nu}^{+} l^{\mu} m^{\nu}=0, \phi_{2}=F_{\mu \nu}^{+} \bar{m}^{\mu} n^{\nu}=0$ and

$$
\phi_{1}=\frac{1}{2} F_{\mu \nu}^{+}\left(l^{\mu} n^{\nu}+\bar{m}^{\mu} m^{\nu}\right)=\frac{m_{+}}{r_{+}^{2}} .
$$

Correspondingly, the only non-vanishing Weyl-NP scalar is

$$
\Psi_{2}=C_{\mu \nu \rho \pi}^{+} l^{\mu} m^{\nu} \bar{m}^{\rho} n^{\pi}=-\frac{m_{+}}{r_{+}^{3}}=\frac{1}{\sigma_{+}} \phi_{1}^{2} .
$$

This simple form of the Weyl doubling formula can also be seen directly from the spinor formulation (cf. [60]).

\subsubsection{Plebanski-Demianski}

The general vacuum type D solution with vanishing cosmological constant [103], as given in [60], is

$$
\begin{aligned}
d s^{2}= & \frac{1}{(1-p q)^{2}} \\
& \times\left[2 i\left(d u+q^{2} d v\right) d p-2\left(d u-p^{2} d v\right) d q+\frac{P(p)}{p^{2}+q^{2}}\left(d u+q^{2} d v\right)^{2}-\frac{Q(q)}{p^{2}+q^{2}}\left(d u-p^{2} d v\right)^{2}\right],
\end{aligned}
$$

with

$$
\begin{aligned}
& P(p)=\gamma\left(1-p^{4}\right)+2 n p-\epsilon p^{2}+2 m p^{3} \\
& Q(q)=\gamma\left(1-q^{4}\right)-2 m q+\epsilon q^{2}-2 n q^{3}
\end{aligned}
$$


where the parameters $m, n, \gamma, \epsilon$ are related to the mass, NUT charge, angular momentum and acceleration (cf. [104]). The self-dual part of the Maxwell two form is given by

$$
F^{+}=\frac{(m-i n)}{2(p+i q)^{2}}\left(i\left(d u+q^{2} d v\right) d p+\left(-d u+p^{2} d v\right) d q\right)
$$

with the anti-self dual part given by the complex conjugate. One has the Weyl doubling formula

$$
C_{\mu \nu \rho \pi}^{+}=\frac{1}{\sigma^{+}} C_{\mu \nu \rho \pi}\left[F^{+}\right],
$$

with $\sigma^{+}=(m-i n)(1-p q)^{4} /(4 i(p+i q)$. Then

$$
\left(F^{2}\right)=\left(\frac{(m-i n)(1-p q)^{2}}{(p+i q)^{2}}\right)^{2}, \quad\left(F^{4}\right)=\frac{1}{4}\left(\frac{(m-i n)(1-p q)^{2}}{(p+i q)^{2}}\right)^{4} .
$$

These are not independent as the eigenvalues of $F^{+}$are repeated - they are $\pm(m-i n)(1-$ $p q)^{2} / 2(p+i q)^{2}$ twice, and hence

$$
\left(C^{+} C^{+}\right)=-\frac{24(m-i n)^{2}(1-p q)^{6}}{(p+i q)^{6}}
$$

is the only independent curvature invariant involving $C^{+}$. Invariants involving $C$ and $C^{*}$ can be written in terms of this and its conjugate.

\subsubsection{Eguchi-Hanson}

The Eguchi-Hanson metric is a vacuum solution with self-dual Weyl curvature. It is given by

$$
d s^{2}=2 d u d v-2 d X d Y+\frac{\lambda}{(u v-X Y)^{3}}(v d u-X d Y)^{2},
$$

with coordinates $(u, v, X, Y)$ and constant $\lambda$. The single-copy (self-dual) Maxwell tensor is

$$
F=\frac{2 \lambda}{(u v-X Y)^{3}}((u v+X Y)(d u \wedge d v-d X \wedge d Y)-2 v Y d u \wedge d X+2 u X d v \wedge d Y) .
$$

This is the single copy tensor discussed in [60], rather than the "mixed" version of [46]. With the Killing vector $K^{\mu}=(u,-v,-X, Y)$ this is given by $F_{\mu \nu}=\left(2 \partial_{[\mu} K_{\nu]}\right)^{+}$. The Weyl curvature is then given by

$$
C_{\mu \nu \rho \sigma}=\frac{1}{\sigma^{+}} C_{\mu \nu \rho \sigma}[F]
$$

with $\sigma^{+}=-\frac{\lambda}{(u v-X Y)}$ and $F$ the Maxwell field (2.32).

As in the case above, the eigenvalues of $F$ are repeated - here they are $\pm m /(u v-X Y)^{2}$ twice. Thus the only independent trace of $F^{n}$ is

$$
\left(F^{2}\right)=\frac{4 \lambda^{2}}{(u v-X Y)^{4}}
$$

and hence the only independent curvature invariant is

$$
\left(C^{2}\right)=\frac{24 \lambda^{2}}{(u v-X Y)^{6}}
$$




\subsubsection{Singly rotating Myers-Perry}

As an example in $D>4$, consider the singly-rotating Myers-Perry metric in the form [105]

$$
\begin{aligned}
d s^{2}= & -\frac{f(x)}{f(y)}(d t+R \sqrt{\nu}(1+y) d \psi)^{2} \\
& +\frac{R^{2}}{(x-y)^{2}}\left[-f(x)\left(g(y) d \psi^{2}+\frac{f(y)}{g(y)} d y^{2}\right)+f(y)^{2}\left(\frac{d x^{2}}{g(x)}+\frac{g(x)}{f(x)} d \phi^{2}\right)\right],
\end{aligned}
$$

with

$$
f(\xi)=1-\xi, \quad g(\xi)=\left(1-\xi^{2}\right)(1-\nu \xi) .
$$

From the Killing vector $K^{\mu}=\partial / \partial t$ form the Maxwell field strength $F_{\mu \nu}=2 \partial_{[\mu} K_{\nu]}$. Then one can check that the Weyl tensor is given by the doubling formula

$$
C_{\mu \nu \rho \pi}=\frac{1}{\sigma} C_{\mu \nu \rho \pi}[F]
$$

with $\sigma=(y-1) /(x-y)$. This satisfies (2.6). One can ask how $K_{\mu}$ might be related to the standard single-copy gauge potential that arises from the Kerr-Schild form of the metric. In the notation of [97], section (E.3), the single-copy potential for the Myers-Perry metrics is $H l_{\mu}$, and it differs from the contravariant vector $K_{\mu}$ obtained from the Killing vector $\partial / \partial \tau$ by a gauge transformation with parameter $\tau$. Thus the two potentials give the same single-copy Maxwell tensor in Kerr-Schild coordinates. When one transforms to the coordinates used in (2.36) above, one obtains that metric, but with the function $g(\xi)$ having no term linear in $\xi$, i.e., with $g(\xi)=1-\xi^{2}+\nu \xi^{3}[106]$. The coefficient of the linear term is purely kinematical [103] and does not affect the vanishing of the Ricci tensor. However, it does appear in the Weyl tensor, and its presence thus affects whether there is a Weyl doubling formula or not. For the function $g[\xi)=1-A \nu \xi-\xi^{2}+\nu \xi^{3}$ it can be checked that only for $A=1$ is there a Weyl doubling formula, which is $(2.38)$.

From $(2.1)$ with $D=5$, the $\left(C^{2}\right)$ and $\left(C^{3}\right)$ invariants are given by the formulæ

$$
\begin{aligned}
& \left(C^{2}\right)=\frac{1}{\sigma^{2}}\left(\frac{15}{8}\left(F^{2}\right)^{2}-\frac{3}{2}\left(F^{4}\right)\right), \\
& \left(C^{3}\right)=\frac{1}{\sigma^{3}}\left(-\frac{23}{2}\left(F^{6}\right)+\frac{81}{8}\left(F^{2}\right)\left(F^{4}\right)-\frac{41}{16}\left(F^{2}\right)^{3}\right) .
\end{aligned}
$$

The recursion relation (2.15) also applies in five dimensions so that the only independent traces are again $\left(F^{2}\right)$ and $\left(F^{4}\right)$. Here they are

$$
\begin{aligned}
\left(F^{2}\right) & =\frac{2\left(-1+L^{2}(1+2 x)\right)(x-y)^{3}}{R^{2}(-1+y)^{3}} \\
\left(F^{3}\right) & =\frac{2\left(1-2 L^{2} x+L^{4}\left(1+2 x+2 x^{2}\right)\right)(x-y)^{6}}{R^{4}(-1+y)^{6}} .
\end{aligned}
$$

The Maxwell field strength here has four distinct eigenvalues — two different pairs with opposite signs, and one zero. 
Turning to the Weyl-NP scalars, the classification of spacetimes in general dimensions via properties of the Weyl tensor has been discussed by Coley, Milson, Pravda and Pravdova (CMPP) in [107, 108] (see also [109, 110]). More recently, the relationship between these classifications has been discussed and compared to a spinor-based analysis in [111], who show that in five dimensions the CMPP and spinor approaches are equivalent. The classification of Maxwell fields is also described there.

We first define a null pentad of five vectors $n^{\mu}, l^{\mu}, m_{i}^{\mu},(i=1,2,3)$, with

$$
n^{\mu} l_{\mu}=-1, \quad m_{i}^{\mu} m_{j \mu}=\delta_{i j}
$$

and all other inner products zero. A convenient set is defined in the appendix A. Now expand a five-dimensional Maxwell field as

$$
F_{\mu \nu}=F_{01} n_{[\mu} l_{\nu]}+\hat{F}_{0 i} n_{[\mu} m_{\nu] i}+\tilde{F}_{1 i} l_{[\mu} m_{\nu] i}+F_{i j} m_{i[\mu} m_{\nu] j} .
$$

There is an analogous expansion for the Weyl tensor. In this type D case the terms with non-zero weights vanish using our pentad choice and we have [107, 108]

$$
C_{\mu \nu \rho \pi}=4 C_{0101} n_{\{\mu} l_{\nu} n_{\rho} l_{\pi\}}+C_{01 i j} n_{\{\mu} l_{\nu} m_{\rho}^{i} m_{\pi\}}^{j}+8 C_{0 i 1 j} n_{\{\mu} m_{\nu}^{i} l_{\rho} m_{\pi\}}^{j}+C_{i j k l} n_{\{\mu} m_{\nu}^{i} l_{\rho} m_{\pi\}}^{j},
$$

with the notation $T_{\{\mu \nu \rho \pi\}}:=\frac{1}{2}\left(T_{[\mu \nu][\rho \pi]}+T_{[\rho \pi][\mu \nu]}\right)$ for a tensor $T_{\mu \nu \rho \pi}$.

The Weyl doubling relationship (2.2) implies that the Weyl coefficients, such as those in (2.43), are given in terms of the analogous Maxwell coefficients, which are given in five dimensions by (2.42). We also saw a simple four-dimensional example of this for the Taub-NUT metric in subsection 2.2.1 above. In five dimensions we find, for example,

$$
C_{0101}=\frac{1}{\sigma}\left(\frac{3}{16} F_{01}^{2}-\frac{1}{8} \hat{F}_{0 i} \tilde{F}_{1 i}-\frac{1}{8} F_{i j} F^{i j}\right) .
$$

For the Myers-Perry metric (2.36) we find the non-zero Maxwell-NP scalars

$$
F_{01}=2 \frac{(x-y)}{R(-1+y)} \sqrt{\frac{\left(-1+L^{2} x\right)(x-y)}{(-1+y)}}, \quad F_{31}=-F_{13}=\frac{L(x-y)}{R(1-y)} \sqrt{\frac{(1+x)(x-y)}{(1-y)}}
$$

and the non-zero independent Weyl-NP scalars

$$
C_{0101}=\frac{\left(-3+L^{2}(1+4 x)\right)(x-y)^{2}}{4 R^{2}(1-y)^{2}}, C_{0131}=-C_{0113}=-\frac{L^{2}(x-y)^{4}(1+x)\left(-1+L^{2} x\right)}{R^{4}(1-y)^{4}} .
$$

(The $C_{i j k l}$ are not independent quantities in five dimensions (cf. also [111] eq. (5.30)). One can then check that for the Myers-Perry metric (2.44) is satisfied using (2.45) and (2.46) and the vanishing of $\hat{F}_{0 i}$ and $\tilde{F}_{1 i}$.

\section{The Gibbons-Hawking metrics}

We will now consider the extension of the Eguchi-Hanson case where there is a self-duality condition on the fields. Here we will find a generalisation of the Weyl doubling formula (2.2), 
where the additional terms are also given in terms of the derivative field strength $F$. The Gibbons-Hawking metric is given by

$$
d s^{2}=\frac{1}{V}\left(d x^{4}+A_{i} d x^{i}\right)^{2}+V d x^{i} d x_{i},
$$

where the fields $V, A^{i}$ are functions of the spatial coordinates $x^{i}, i=1,2,3$, and are related by

$$
\nabla V=\nabla \times A .
$$

This equation implies $V$ is harmonic. It may thus be solved by a superposition of harmonic functions with arbitrary centres. The two centre solution can be shown to be equivalent to the Eguchi-Hanson solution, after a coordinate transformation. From the Killing vector $K^{\mu}=(0,0,0,1)$ we can form the anti-self-dual field strength

$$
F_{\mu \nu}=2 \partial_{[\mu} K_{\nu]}
$$

satisfying $F_{\mu \nu}^{*}=\frac{1}{2} \epsilon_{\mu \nu \rho \sigma} F^{\rho \sigma}=-F_{\mu \nu}$ with $\sigma=1 / V$ via (2.6). We find in this case that a simple Weyl doubling formula of the form of equation (2.2) does not hold. To explore this further, note that as the Weyl curvature is anti-self-dual, it has only five independent components, which may be taken to be the components of the symmetric traceless matrix $3 \times 3$ matrix $C_{i 4 j 4}$. One can express this using the following three anti-self-dual two forms. First note that the vierbein one-forms are given by

$$
\begin{aligned}
e^{4} & =\frac{1}{\sqrt{V}}\left(d x^{4}+A_{i} d x^{i}\right), \\
e^{i} & =\sqrt{V} d x^{i} .
\end{aligned}
$$

Then the two forms are given by

$$
\Sigma^{i}=e^{4} e^{i}+\frac{1}{2} \epsilon^{i j k} e^{j} e^{k}
$$

We now solve the following equation for $\hat{\Omega}^{i}$ :

$$
d \Sigma^{i}+\epsilon^{i j k} \hat{\Omega}^{j} \Sigma^{k}=0 .
$$

This implies that $\hat{\Omega}^{i}$ is given by the anti-self-dual part of the spin connection, $\omega_{\nu}^{\mu}$ and thus

$$
\hat{\Omega}^{i}=\omega^{i 4}-\frac{1}{2} \epsilon_{j k}^{i} \omega^{j k} .
$$

The curvature can then be constructed directly from the curvature of $\hat{\Omega}^{i}$ and is given by

$$
\hat{C}^{i}=d \hat{\Omega}^{i}+\frac{1}{2} \epsilon^{i j k} \hat{\Omega}^{j} \hat{\Omega}^{k}=\hat{C}^{i j} \Sigma^{j},
$$

where we have introduced $\hat{C}^{i j}$ encoding the Weyl tensor. We now use Cartan's first structure equation to calculate the connection one forms for the vierbeins as follows

$$
\omega^{4 i}=V^{-3 / 2}\left(-\frac{1}{2} \partial^{i} V e^{4}+\partial^{[i} A^{j]} e_{j}\right), \quad \omega^{i j}=V^{-3 / 2}\left(\frac{1}{2} \partial^{j} V e^{i}-\partial^{[i} A^{j]} e^{4}\right) .
$$


Then, expanding both the Maxwell field strength and the curvature in terms of the two forms $\Sigma^{i}$ we find the following doubling formula

$$
\begin{aligned}
F & =\alpha_{i} \Sigma^{i}, \\
\hat{C}^{i} & =\left(V \alpha_{i} \alpha_{j}-\partial_{i} \alpha_{j}\right)^{T} \Sigma^{j},
\end{aligned}
$$

where the superscript $T$ means to take the traceless part of the expression within the brackets and

$$
\alpha_{i}=\frac{1}{V^{2}} \partial_{i} V .
$$

In terms of the Weyl tensor we have the relation $\hat{C}_{i j}=-2 C_{i 4 j 4}$, with the other components of the Weyl tensor related to these by the anti-self-duality condition. Equation (3.10) may be viewed a generalised form of Weyl doubling, where the quadratic, algebraic terms involving the gauge field are supplemented by terms depending on the derivatives of the gauge field. Thus we see that the general class of gravitational instantons in four dimensions satisfies a generalised Weyl doubling formula.

An explicitly covariant version of this formula can be found as follows. There is the identity

$$
C_{\mu \nu \rho \sigma}=-2 V\left(K_{\mu} K^{\lambda} C_{\nu \lambda \rho \sigma}+K_{\rho} K^{\lambda} C_{\sigma \lambda \mu \nu}-2 g_{\rho \mu} C_{\nu \lambda \pi \sigma} K^{\lambda} K^{\pi}\right)_{[\mu \nu][\rho \sigma]}
$$

where the notation $[\mu \nu][\rho \sigma]$ means to antisymmetrise the expression within the preceding brackets in $\mu, \nu$ and separately in $\rho, \sigma$. The above relation follows from the fact that $C^{*}=-C$ and $K^{2}=-\sigma=\frac{1}{V}$. To see this, define the three expressions on the righthand side of (3.12), including the antisymmetrisations, as $C_{L}+C_{R}+C_{g}$. Then, taking the (left) dual of each by contracting with $\frac{1}{2} \epsilon^{\alpha \beta \mu \nu}$, one finds that $\left(C_{L}+C_{R}+C_{g}\right)^{*}=$ $\left(-C-C_{L}\right)+\left(C_{R}\right)+\left(C_{L}-C_{R}\right)=-C=C^{*}$.

Equation (3.12) is equivalent to

$$
C_{\mu \nu \rho \sigma}=V\left(K_{[\mu} \nabla_{\nu]} F_{\rho \sigma}+K_{[\rho} \nabla_{\sigma]} F_{\mu \nu}\right)^{T}
$$

where the notation $(\ldots)^{T}$ means to subtract all traces. This can be re-expressed as the doubling formula

$$
C_{\mu \nu \rho \sigma}=V C_{\mu \nu \rho \sigma}[F]+V\left(2 \nabla_{\rho}\left(K_{\mu} F_{\nu \sigma}\right)-\nabla_{\mu}\left(K_{\nu} F_{\rho \sigma}\right)-\nabla_{\rho}\left(K_{\sigma} F_{\mu \nu}\right)-K_{\mu} \nabla_{\nu} F_{\rho \sigma}\right)_{[\mu \nu][\rho \sigma]}^{T} .
$$

where the notation $(\ldots)^{T}$ means to subtract all traces.

As the two-centred solution for the Gibbons-Hawking (GH) metric (3.1) is equivalent to the Eguchi-Hanson (EH) metric (2.31) via a coordinate transformation one may wonder why there is a simple doubling formula for the $\mathrm{EH}$ metric described earlier, but not for the more general multi-centred GH metrics. This special case can be understood by noting that for spherical polar coordinates the map from the EH metric to the two-centred GH metric (see [112] for example) interchanges the periodic "time" coordinate and the azimuthal angle $\phi$. Mapping to the two-centred GH case, the Killing vector which gives a simple 
Weyl doubling formula via the anti-self-dual part of the Maxwell field $2 \partial_{[\mu} K_{\nu]}$ is then $K^{\mu}=\frac{\partial}{\partial \phi}$. If one constructs the Maxwell field via the Killing vector $K^{\mu}=\frac{\partial}{\partial t}$ then one obtains the formulæ in the analysis above for this particular potential. In the generic multicentre case there is only the latter Killing vector, leading to the above analysis. (In the multi-centre case where the centres are all at different sites along the $z$-axis, the additional Killing vector is present of course - here we find evidence from a numerical analysis that the simpler Weyl doubling formula continues to hold.)

\section{An eight-dimensional example with $\operatorname{Spin}(7)$ holonomy}

The discussion above used features of the Gibbons-Hawking metrics which arise from the underlying self duality relation (3.2) which expresses the anti-self-duality of the field strength of the four vector gauge field $\left(V, A^{i}\right)$. In higher dimensions, manifolds of special holonomy are examples where there are more novel duality conditions satisfied by the curvature, and two forms on the manifold can similarly be restricted to have duality properties determined by the canonical four-form defined by the special holonomy group (a pioneering paper on this is [113]; see [114] for a review relevant to string theory). However, in general the formula (2.1) does not preserve duality properties. This means that if a two-form $F$ transforms in a certain representation of the holonomy group then this does not imply that $C[F]$ will as well (in each pair of indices). Furthermore, projecting $C[F]$ onto appropriate representations does not in general preserve the algebraic symmetries needed to relate it to a Weyl tensor. While these conditions may appear quite restrictive, we have found an example of an eight-dimensional manifold with $\operatorname{Spin}(7)$ holonomy for which Weyl doubling does work. We will use the conventions of [115] in what follows.

Begin with the metric [116]

$$
d s^{2}=V^{-3 / 2}\left(d x^{8}+A_{i} d x^{i}\right)^{2}+V^{1 / 2} d x^{i} d x_{i},
$$

where here the indices $i, j, \ldots$ run from 1 to 7 , and $a, b, \ldots$ and $\mu, \nu, \ldots$ run from 1 to 8 . The fields $\left(A_{i}, V\right)$ are functions of the spatial coordinates $x^{i}$ only. The $\operatorname{spin}(7)$ four-form $\phi_{a b c d}$ has the following non-zero orthonormal frame components

$$
\begin{aligned}
{[1256]=[1278]=[3456] } & =[3478]=[1357]=[2468]=[1234]=[5678]=1, \\
{[1368] } & =[2457]=[1458]=[1467]=[2358]=[2367]=-1,
\end{aligned}
$$

where $[a b c d]$ means $\phi_{a b c d}$. The four-form satisfies $\phi^{2}=4 \phi+12$ and the projectors of a two form onto the "self-dual" $\mathbf{7}$ and "anti self-dual" $\mathbf{2 1}$ representations of $\operatorname{Spin}(7)$ are given by

$$
P_{7}=\frac{1}{4}\left(1+\frac{1}{2} \phi\right), \quad P_{21}=\frac{3}{4}\left(1-\frac{1}{6} \phi\right) .
$$

Define the acht-beins

$$
\begin{aligned}
e^{a} & =\left(e^{i}, e^{8}\right) \\
e^{i} & =V^{1 / 4} d x^{i} \\
e^{8} & =V^{-3 / 4}\left(d x^{8}+A_{i} d x^{i}\right)
\end{aligned}
$$


Then the four form

$$
\phi=\frac{1}{24} \phi_{a b c d} e^{a} e^{b} e^{c} e^{d}
$$

is closed provided that the fields $\left(A_{i}, V\right)$ satisfy the constraints $\left(F_{i j}:=\partial_{i} A_{j}-\partial_{j} A_{i}\right.$ and $\left.V_{i}:=\partial_{i} V\right)$

$$
V_{[i} \phi_{j k l m]}-2 F_{[i j} \phi_{k l m] 8}=0 .
$$

These 21 equations can be solved in terms of 7 independent quantities. For example, choosing the independent variables to be $\left(F_{37}, F_{45}, F_{46}, F_{47}, F_{56}, F_{57}, F_{67}\right)$ the other expressions are given by

$$
\begin{aligned}
F_{12} & =F_{56}, F_{13}=F_{57}, F_{14}=-F_{67}, F_{15}=-F_{37}, F_{16}=F_{47}, F_{17}=-F_{46}, F_{23}=-F_{67}, \\
F_{24} & =-F_{57}, F_{25}=F_{47}, F_{26}=F_{37}, F_{27}=-F_{45}, F_{34}=F_{56}, F_{35}=-F_{46}, F_{36}=F_{45}, \\
V_{1} & =-2 F_{45}, V_{2}=2 F_{46}, V_{3}=2 F_{47}, V_{4}=-2 F_{37}, V_{5}=2 F_{67}, V_{6}=-2 F_{57}, V_{7}=2 F_{56} .
\end{aligned}
$$

It can be checked that $\phi$ is also covariantly constant when these conditions are met. In fact, taking various linear combinations of the constraints (4.6) one finds that $\partial_{i} \partial_{j} V=0$ for all $i, j$ so that $V$ is linear in the coordinates $x^{i}$ and the correction term is trivial. This also means that all the electric $V_{i}$ and magnetic $F_{i j}$ components of the gravitational field are constants. Now consider the construction of the Maxwell field. From the Killing vector $K^{\mu}=\delta^{\mu 8}$ we can again form the gauge field strength

$$
F_{\mu \nu}=2 \partial_{[\mu} K_{\nu]}
$$

This field is anti self-dual i.e. in the $\mathbf{2 1}$ representation, as $P_{7} F=0$. Define the anti self-dual two forms

$$
\Sigma_{-}^{i j}=P_{21} e^{a} \wedge e^{b} .
$$

These satisfy the orthonormality conditions

$$
\Sigma_{-}^{a b \mu \nu} \Sigma_{-c d \mu \nu}=4\left(P_{21}\right)_{c d}^{a b}, \quad \Sigma_{-}^{a b \mu \nu} \Sigma_{-a b \rho \sigma}=\left(P_{21}\right)_{\rho \sigma}^{\mu \nu} .
$$

We can expand the gauge field two form in the basis of these as

$$
F_{\mu \nu}=\frac{1}{2} \alpha_{a b} \Sigma_{-\mu \nu}^{a b},
$$

with $\alpha_{a b}=\frac{1}{2} \Sigma_{-a b}^{\mu \nu} F_{\mu \nu}$ which can be written as

$$
\alpha_{i j}=-\frac{1}{2 V^{2}} \phi_{8 i j k} V_{k}, \quad \alpha_{i 8}=-\frac{3}{2 V^{2}} V_{i} .
$$

The metric is Ricci-flat and it can be checked that the Weyl tensor $C_{\mu \nu \rho \sigma}$ satisfies $\left(P_{7} C\right)_{\mu \nu \rho \sigma}=0$, with $P_{7}$ acting either on the first or second pair of indices of the Weyl tensor. The tangent space components of the Weyl tensor can be expressed purely in terms of the constants $V_{i}$. Let us then consider if these can be given as a doubling formula based on a two form such as (4.11) above. We will generalise this expression slightly and consider the "twisted" two form with components

$$
\alpha_{i j}=-\frac{b}{2 V^{2}} \phi_{8 i j k} V_{k}, \quad \alpha_{i 8}=-\frac{3 a}{2 V^{2}} V_{i},
$$


for some constants $a, b$. This two form will be anti self-dual if $a=b$. The natural expression to consider for a doubling formula is one based on (2.1) in eight dimensions, $C_{a b c d}[\alpha]$ with $\alpha$ given by (4.13). In four dimensions the formula (2.1) preserves duality - if $F_{\mu \nu}$ is self-dual or anti self-dual then $C_{\mu \nu \rho \sigma}[F]$ is also (in both pairs of indices). But this is not true in higher dimensions and in this eight-dimensional case, in order to seek to match the Weyl tensor this expression must be projected onto the $\mathbf{2 1}$ on the left and right pairs of indices. Upon doing this we find that it is not possible to both preserve the duality conditions and the symmetry and trace properties of a Weyl-type tensor unless $a$ and $b$ satisfy the condition $23 a^{2}-42 a b+27 b^{2}=0$. We will write a solution of this as $a=\gamma b$ with $\gamma$ a particular complex number. In this case we find that there is a doubling formula for the Weyl tensor

$$
C_{a b c d}=\lambda C_{a b c d}^{21}[\alpha],
$$

where the superscript 21 on $C$ indicates that both pairs of indices are to be projected into the $\mathbf{2 1}$ representation. The proportionality constant is $\lambda=-23 V^{3 / 2} /\left(32 b^{2}(\gamma+1)\right)$. The field $\alpha$ here is not anti self-dual as we have noted, but it can be checked that its anti self-dual part is proportional to the Maxwell tensor (4.11), and that the corresponding field strength $F_{\mu \nu}$ satisfies the Maxwell field equations.

\section{$5 \quad$ Reissner-Nordström and Born-Infeld}

In the sections above, we studied examples where the gauge field arises from intrinsic properties of the spacetime. We now turn to study situations where there is an independent gauge field defined on the spacetime, in order to see if there is a doubling relationship whereby the gauge fields in the gravity-gauge system give formulæ for the full spacetime curvature - determining the Ricci tensor via the Einstein equations as usual but in addition fixing the Weyl tensor via a doubling formula. We will call this "self-doubling" for simplicity.

\subsection{Reissner-Nordström}

As a first case, consider the Reissner-Nordström theory in $D$ dimensions, with metric

$$
d s^{2}=-f(r) d t^{2}+f^{-1}(r) d r^{2}+r^{2} d \Omega_{D-2}^{2},
$$

with

$$
f(r)=1-\frac{2 M}{r^{D-3}}+\frac{Q^{2}}{r^{2(D-3)}},
$$

$d \Omega_{D-2}^{2}$ the metric on the $(D-2)$-dimensional sphere, and $M$ and $Q$ the mass and charge respectively.

This theory is self-doubling in the sense that the gauge field already present in the theory provides the basis for the doubling. This can be seen as follows - the gauge field one form is

$$
A=-\frac{Q}{r^{D-3}} d t
$$


satisfying the field equations $\left(F_{\mu \nu}=2 \partial_{[\mu} A_{\nu]}\right)$

$$
\begin{aligned}
\nabla^{\mu} F_{\mu \nu} & =0, \\
R_{\mu \nu}-\frac{1}{2} g_{\mu \nu} R & =\frac{D-2}{D-3} T_{\mu \nu},
\end{aligned}
$$

with the usual stress tensor

$$
T_{\mu \nu}=F_{\mu} \cdot F_{\nu}-\frac{1}{4} g_{\mu \nu} F \cdot F
$$

We find the Weyl doubling formula

$$
C_{\mu \nu \rho \sigma}=\Lambda(r) C_{\mu \nu \rho \sigma}[F]
$$

where on the right-hand side of this equation $C_{\mu \nu \rho \sigma}[F]$ is given by the formula (2.1) and the coefficient is

$$
\Lambda(r)=\frac{2(D-2)}{3(D-3)^{2}}\left((2 D-5)-(D-1) \frac{M r^{D-3}}{Q^{2}}\right) .
$$

This result has been checked up to $D=10$ but there are no reasons why this would not hold for all dimensions given the structure of the metric. Notice that one can take the extremal limit $Q \rightarrow M$ directly in the equations above.

\subsection{Born-Infeld}

The discussion above may be generalised to the Born-Infeld theory in any dimension. The Lagrangian is

$$
L=\sqrt{g} R+\frac{1}{\lambda^{2}}(\sqrt{g}-\sqrt{|\operatorname{det}(g+\lambda F)|})
$$

with $F_{\mu \nu}$ the Maxwell field and $\lambda$ a constant. The solution with electric charge $Q$ in four dimension is given by (e.g. [117])

$$
\begin{aligned}
& d s^{2}=-\left(1-\frac{2 m(r)}{r^{2}}\right) d t^{2}+\left(1-\frac{2 m(r)}{r^{2}}\right)^{-1} d r^{2}+r^{2}\left(d r^{2}+d \Omega_{2}^{2}\right) \\
& F_{t r}=-F_{r t}=\frac{Q}{\sqrt{r^{4}+\lambda^{2} Q^{2}}}
\end{aligned}
$$

with the other components of $F$ vanishing. The function $m(r)$ is fixed by the metric equation of motion, and satisfies

$$
m^{\prime}(r)=\frac{1}{\lambda^{2}}\left(\sqrt{r^{4}+\lambda^{2} Q^{2}}-r^{2}\right)
$$

the solution of which is given by equation (5.15) below with $D=4$. We also define the tensor

$$
G^{\mu \nu}=-\frac{2}{\sqrt{g}} \frac{\partial L}{\partial F_{\mu \nu}}
$$


which satisfies the (non-linear in $F$ ) field equations $\nabla^{\mu} G_{\mu \nu}=0$. For the solution $F$ in (5.9) this is given simply by $G_{t r}=-G_{r t}=\frac{Q}{r^{2}}$ with other components vanishing. It is straightforward to confirm that the Weyl tensor satisfies the equation

$$
C_{\mu \nu \rho \sigma}=\Lambda_{4}(r) C_{\mu \nu \rho \sigma}[G]
$$

with

$$
\Lambda_{4}(r)=-\frac{2 r}{3 Q^{2}}\left(6 m(r)-4 r m^{\prime}(r)+r^{2} m^{\prime \prime}(r)\right) .
$$

This discussion is easily generalised to $D$ dimensions. The metric is as in (5.9) with $d \Omega_{2}^{2} \rightarrow d \Omega_{D-2}^{2}$ with the function $m(r)$ satisfying

$$
m^{\prime}(r)=\frac{1}{\lambda^{2}}\left(\sqrt{r^{2(D-2)}+\lambda^{2} Q^{2}}-r^{D-2}\right) .
$$

This is solved by

$$
\begin{aligned}
\lambda^{2} m(r)= & \lambda^{2} m+\frac{r^{D-1}}{D-1}-\frac{r \sqrt{\lambda^{2} Q^{2}+r^{2(D-2)}}}{D-1} \\
& +\frac{\lambda^{2} Q^{2}(D-2)}{(D-1)(D-3) r^{D-3}}{ }_{2} F_{1}\left[\frac{1}{2}, \frac{D-3}{2(D-2)}, 1+\frac{D-3}{2(D-2)},-\frac{\lambda^{2} Q^{2}}{r^{2(D-2)}}\right] .
\end{aligned}
$$

for constant $m$. Note that $m(r) \rightarrow m$ in the limit $r \rightarrow 0$.

One has the result

$$
C_{\mu \nu \rho \sigma}=\Lambda(r) C_{\mu \nu \rho \sigma}[G]
$$

with

$$
\Lambda(r)=-\frac{2 r^{D-3}}{3 Q^{2}}\left((D-1)(D-2) m(r)-2(D-2) r m^{\prime}(r)+r^{2} m^{\prime \prime}(r)\right)
$$

and the field $G$ in $D$ dimensions given by

$$
G_{t r}=-G_{r t}=\frac{Q}{r^{D-2}},
$$

with other components vanishing. Notice that $G_{\mu \nu}$ is a function of the Maxwell field $F_{\mu \nu}$ via the relation (5.11). The result (5.16) leads to curvature singularities as $r \rightarrow 0$ which are not present in the field $F_{\mu \nu}$.

This Born-Infeld solution reduces to the Reissner-Nordström model in the section above in the limit as $\lambda \rightarrow 0$, using the expansion

$$
\operatorname{det}\left(\delta^{\mu}{ }_{\nu}+\lambda F^{\mu}{ }_{\nu}\right)=1-\frac{1}{2} \lambda^{2}\left(F^{2}\right)-\frac{1}{4} \lambda^{4}\left(\left(F^{4}\right)-\frac{1}{2}\left(F^{2}\right)^{2}\right)+o\left(\lambda^{6}\right),
$$

where the brackets in $\left(F^{2}\right),\left(F^{4}\right)$ indicate matrix traces of powers of $F^{\mu}{ }_{\nu}$. It can be checked that the coefficient in (5.17) reduces in this limit to that in (5.7).

The formula (5.16) enables one to easily find invariants, for example

$$
C^{\mu \nu \rho \sigma} C_{\mu \nu \rho \sigma}=\frac{4(D-3)}{(D-1) r^{2(D-1)}}\left((D-1)(D-2) m(r)-2(D-2) r m^{\prime}(r)+r^{2} m^{\prime \prime}(r)\right)^{2} .
$$


Note that this diverges as $1 / r^{2(D-1)}$ as $r \rightarrow 0$, although the non-zero Maxwell field strength component $F_{t r}=Q / \sqrt{r^{2 D-4}+\lambda^{2} Q^{2}}$ does not. The divergence comes from the traces of the field $G_{\mu \nu}$ which appear when squaring (5.16). (A discussion of the four dimensional Born-Infeld theory appeared recently [118] and the $D=4$ version of (5.20) appeared there, although their equivalent of $m(r)$ satisfies a different equation.)

\section{Brane solutions}

It is natural to conjecture that BPS brane solutions in supergravity (we will follow the conventions of [119] in this section) might satisfy Weyl doubling. The $(p+1)$-forms that minimally couple to the $p$-brane provide a potential from which one can construct a $(p+2)$ form field strength. This field strength can then be used in the formula for the Weyl curvature, as well as determining the Ricci curvature via the field equations and stress tensor. We consider here the cases of $p$-branes where the scalar fields play no role.

\subsection{String in five dimensions}

A simple example is the string in five dimensions. The metric and two-form are given by

$$
\begin{aligned}
d s^{2} & =H^{-1}(r)\left(-d x_{1}^{2}+d x_{2}^{2}\right)+H^{2}(r)\left(d r^{2}+r^{2}\left(d \theta^{2}+\sin ^{2} \theta d \phi^{2}\right)\right), \\
B_{\mu \nu} & =\sqrt{3} \epsilon_{\mu \nu} H^{-1}(r),
\end{aligned}
$$

with $H(r)=1+k / r$ and indices $\mu, \nu=1,2$. The three-form field strength $F=d B$, obeys $\nabla^{M} F_{M N P}=0$ and the metric field equation

$$
G_{M N}=\frac{1}{4}\left(F_{M} \cdot F_{N}-\frac{1}{6} g_{M N} F \cdot F\right) .
$$

For the $p$-forms $F$ discussed in the subsections below the equivalent equation is

$$
G_{M N}=\frac{1}{2(p-1) !}\left(F_{M} \cdot F_{N}-\frac{1}{2 p} g_{M N} F \cdot F\right) .
$$

We will now define the two-forms $\left(F_{\mu}\right)_{N P}=\left(K_{\mu}\right)^{M} F_{M N P}(\mu=1,2)$ where the Killing vectors $K_{\mu}$ correspond to translations in the $x^{\mu}$ directions in the string world sheet. Note that $K_{1}^{2}=H^{-1}(r)=-K_{2}^{2}$. We then find that the following Weyl doubling formula may be constructed from the three-form field strength $F$ and the two-form field strengths $F_{\mu}$

$$
C_{M N P Q}=-\frac{(k+4 r)}{6 k} C_{M N P Q}[F]-\frac{r H(r)}{3 k}\left(-C_{M N P Q}\left[F_{1}\right]+C_{M N P Q}\left[F_{2}\right]\right) .
$$

This can also be written as

$$
C_{M N}{ }^{P Q}=-\frac{1}{6} C_{M N}{ }^{P Q}[F]+\Sigma_{1}(r) T_{M N}{ }^{P Q},
$$

where $\Sigma_{1}(r)=\frac{k}{r^{3} H^{4}}$. We note that $\Sigma_{1}(r)$ is proportional to the inverse of the volume of the transverse sphere. In this coordinate system all of the non-zero components of the Weyl tensor take the form (up to sign) $C_{M N}{ }^{M N}$. The only non-zero components of $C_{M N}{ }^{P Q}[F]$ 
and the tensor $T_{M N} P Q$ are similarly when $(P, Q)=(M, N)$ (or $\left.(N, M)\right)$. In this case the $T_{M N} P Q$ are given by $(0,1,-1 / 2,-1,2)$ for $(M N)=(12, \mu 3, \mu \bar{m}, 3 \bar{m}, 45)$ respectively $(\bar{m}=4,5)$, and components related to these by the antisymmetry in $M, N$. Notice thus that the tensor $T$ vanishes when all its components are along the world-volume, and so from (6.5) we see that on the world-volume there is a simple Weyl doubling formula, and this is corrected off the world volume by a tensor that takes a simple form.

In [120] the Kerr-Schild formulation was investigated in the case where there is both a metric and a Kalb-Ramond field, using doubled geometry. It was found that this involved two null vectors with the metric and $B$ field involving the symmetric and anti-symmetric product of these vectors. It is natural then to expect that a single copy in this case should involve two Maxwell gauge fields $A$ and $\bar{A}$. For the five-dimensional case under consideration here, one can see that the $B$ field in (6.1) is given by

$$
B_{M N}=2 \sqrt{3} H A_{[M} \bar{A}_{N]},
$$

with

$$
\begin{aligned}
& A_{M}=\left(H^{-1}, 0,0,0,0\right), \\
& \bar{A}_{M}=\left(0, H^{-1}, 0,0,0\right) .
\end{aligned}
$$

This generates a formula for $F_{M N P}$ in terms of $A_{M}$ and $\bar{A}_{M}$.

One avenue suggested by this work is to develop a Weyl doubling formula for DFT. Along these lines, one may view an expression of the formula (6.5) as a Weyl "doubling" in terms of the two Maxwell fields in the formalism of [120]. Inserting (6.6) into (6.5) would then give an expression for the Weyl tensor in terms of an expression quartic in fields and two derivatives. (Note that the expression (6.6) is in the usual double copy where the vector fields in that formalism live in flat space.)

\subsection{M2 brane}

For the M2 brane in eleven dimensions, the metric and non-zero three-form potential components are given by

$$
\begin{aligned}
d s^{2} & =H^{-2 / 3}(r)\left(-d x_{1}^{2}+d x_{2}^{2}+d x_{3}^{2}\right)+H^{1 / 3}(r)\left(d r^{2}+r^{2} d \Omega_{7}^{2}\right), \\
A_{\mu \nu \rho} & =\epsilon_{\mu \nu \rho} H^{-1}(r),
\end{aligned}
$$

with $H(r)=1+k / r^{6}$ and indices $\mu, \nu, \ldots=1,2,3$, and $m, n, \ldots=4, \ldots, 11$ (with $M, N, \ldots=1, \ldots, 11) . r$ is the radial coordinate in the eight-dimensional transverse space. The four-form field strength $F=d A$ has non-zero components $F_{1234}=-6 k H^{-2} / r^{7}$ and obeys $\nabla^{M} F_{M N P Q}=0$ and (6.3) with $p=4$.

Again we define the tensors $\left(F_{\mu}\right)_{M N P}=\left(K_{\mu}\right)^{Q} F_{Q M N P}$ where the Killing vectors $K_{\mu}$ correspond to translations in the $x^{\mu}$ directions in the world-volume. The Weyl doubling formula can then be constructed as follows:

$C_{M N P Q}=\frac{\left(k+4 r^{6}\right)}{36 k} C_{M N P Q}[F]-\frac{r^{6} H(r)^{2 / 3}}{3 k}\left(-C_{M N P Q}\left[F_{1}\right]+C_{M N P Q}\left[F_{2}\right]+C_{M N P Q}\left[F_{3}\right]\right)$. 
This simplifies along the world-volume as in the string case above, with the analogue of (6.5) being

$$
C_{M N}{ }^{P Q}=\frac{1}{36} C_{M N}{ }^{P Q}[F]+\Sigma_{2}(r) T_{M N} P Q,
$$

where here $\Sigma_{2}(r)=\frac{2 k H^{-7 / 3}}{r^{8}}$ and the object $T_{M N} P Q$ has non-zero components $(0,7,-1,-3,1)$ for $(P Q)=(M N)=(\mu \nu, \mu 4, \mu \bar{m}, 4 \bar{m}, \bar{m} \bar{n})$ respectively, with $\bar{m}, \bar{n}=$ $5, \ldots, 11$. Again we note that $\Sigma_{2}(r)$ is proportional to the inverse of the volume of the transverse sphere.

Whilst a Kerr-Schild type formulation based on exceptional geometry (see [121] for a review) is not yet available, we note that for SL(5) the 10 representation reduces to $4+6$ in four dimensions, representing a vector and two-form which are expected to be fundamental to this. Thus one might expect that a single copy in this case might involve a Maxwell gauge field $A_{M}$ and two-form $A_{M N}$, with the $A_{M N P}$ field.

\subsection{D3 brane}

For the D3 brane in ten dimensions, the metric and non-zero four-form potential components are given by

$$
\begin{aligned}
d s^{2} & =H^{-1 / 2}(r)\left(-d x_{1}^{2}+d x_{2}^{2}+d x_{3}^{2}+d x_{4}^{2}\right)+H^{1 / 2}(r)\left(d r^{2}+r^{2} d \Omega_{5}^{2}\right), \\
A_{\mu \nu \rho \sigma} & =\epsilon_{\mu \nu \rho \sigma} H^{-1}(r),
\end{aligned}
$$

with $H(r)=1+k / r^{4}$ and indices $\mu, \nu \ldots=1,2,3,4$, and $m, n, \ldots=5 \ldots, 10$ (and $M, N, \ldots=1, \ldots, 10) . r$ is the radial coordinate in the six-dimensional transverse space. We will need the self-dual five-form field strength $F=\frac{1}{2}\left(d A+(d A)^{*}\right)$. As above, we define the tensors $\left(F_{\mu}\right)_{M N P Q}=\left(K_{\mu}\right)^{R} F_{R M N P Q}$ where the Killing vectors $K_{\mu}$ correspond to translations in the $x^{\mu}$ directions in the world-volume. The Weyl doubling formula is then found to be

$$
\begin{aligned}
C_{M N P Q}= & \frac{r^{4}}{6 k} C_{M N P Q}[F] \\
& -\frac{2 r^{4} H(r)^{1 / 2}}{3 k}\left(-C_{M N P Q}\left[F_{1}\right]+C_{M N P Q}\left[F_{2}\right]+C_{M N P Q}\left[F_{3}\right]+C_{M N P Q}\left[F_{4}\right]\right)
\end{aligned}
$$

This also shows that the Weyl tensor vanishes for components along the world-volume. To see this, one has the equivalent expression

$$
C_{M N}{ }^{P Q}=\Sigma_{3}(r) T_{M N}{ }^{P Q},
$$

where $\Sigma_{3}(r)=\frac{k H^{-5 / 2}}{r^{6}}$ and the object $T_{M N}{ }^{P Q}$ has non-zero components $(0,5,-1,-4,2)$ for $(P Q)=(M N)=(\mu \nu, \mu 5, \mu \bar{m}, 5 \bar{m}, \bar{m} \bar{n})$ respectively, with $\bar{m}, \bar{n}=6, \ldots, 10$. As in the cases above, $\Sigma_{3}(r)$ is proportional to the inverse of the volume of the transverse sphere.

\subsection{M5 brane}

There is a similar story for the M5 brane in eleven dimensions. The metric and non-zero 4 -form field strength components are given by

$$
\begin{aligned}
d s^{2} & =H^{-1 / 3}(r) \eta_{\mu \nu} d x^{\mu} d x^{\nu}+H^{2 / 3}(r)\left(d r^{2}+r^{2} d \Omega_{4}^{2}\right), \\
F_{891011}^{(4)} & =3 k \sin ^{3}(\theta) \sin ^{2}(\phi) \sin \left(\psi_{1}\right),
\end{aligned}
$$


with $F^{(4)}$ antisymmetrised, $H(r)=1+k / r^{3}$, world-volume coordinates $x^{\mu}(\mu=1, \ldots 6)$, and transverse coordinates $r$ and spherical polars $\left(\theta, \phi, \psi_{1}, \psi_{2}\right)$.

The fivebrane magnetically couples to the three form $C_{3}$ which means we will need to use the magnetic dual field strength given by the seven-form, $F={ }^{*} F^{(4)}$. Then define $\left(F_{\mu}\right)_{N P Q R S T}=\left(K_{\mu}\right)^{M} F_{M N P Q R S T}(\mu=1,2, \ldots, 6)$ with the Killing vectors $K_{\mu}$ corresponding to translations in the $x^{\mu}$ directions in the world-volume. The Weyl doubling formula is then

$$
\begin{aligned}
36 C_{M N P Q}= & -\frac{1}{60} C_{M N P Q}[F]+\frac{r^{3}}{3 k} C_{M N P Q}[F] \\
& -\frac{r^{3}}{2 k} H(r)^{1 / 3}\left(-C_{M N P Q}\left[F_{1}\right]+C_{M N P Q}\left[F_{2}\right]+\cdots+C_{M N P Q}\left[F_{6}\right]\right) .
\end{aligned}
$$

This is equivalent to

$$
36 C_{M N}^{P Q}=-\frac{1}{60} C_{M N}^{P Q}[F]+\Sigma_{5}(r) T_{M N}^{P Q},
$$

where here $\Sigma_{5}(r)=\frac{18 k H^{-8 / 3}}{r^{5}}$ and the object $T_{M N} P Q$ has non-zero components $(0,4,-1,-6,4)$ for $(P Q)=(M N)=(\mu \nu, \mu 7, \mu \bar{m}, 7 \bar{m}, \bar{m} \bar{n})$ respectively, with $\bar{m}, \bar{n}=$ $8,9,10,11$. We see again there is a simple doubling formula for the Weyl components along the world-volume directions and that $\Sigma_{5}(r)$ is proportional to the inverse of the volume of the transverse sphere.

In all of the cases above, for a brane with $V$-dimensional world-volume in $D$ dimensions, and $T=D-V$ transverse dimensions, the components of the tensor $T_{M N}{ }^{M N}$ are proportional to $(0, T-1,-1,-V, 2 V /(T-2))$ for $(P Q)=(M N)=(\mu \nu, \mu r, \mu \bar{m}, r \bar{m}, \bar{m} \bar{n})$ respectively, in the notation used above. Given that the components along the world-volume vanish, this follows from the tracelessness condition on the Weyl tensor. The powers or $r$ and $H$ in the coefficients $\Sigma$ are equal to $-T$ and that appearing in the inverse of the volume of the transverse sphere respectively. Similarly, the powers of $r$ and $H$ in the doubling formulæ above have common expressions: $r^{T-2}$ and the inverse of the power of $H$ which appears in the world-volume metric.

\section{Discussion}

We described Weyl doubling as the writing of the Weyl tensor (up to a scalar factor) in terms of a quadratic expression in an Abelian field strength obeying Maxwell's equations in a curved background. The curved background distinguishes these results from the usual double copy originating in scattering amplitudes and more recently classical solutions using the Kerr-Schild form. This phenomenon of Weyl doubling was found in a variety of solutions in different dimensions. The purely intrinsic case is where a Killing vector on the manifold is used to define a potential from which the field strength is derived. The Weyl tensor is given by the formula (2.1). We showed that the metrics for which the Weyl doubling formula (2.1) applies fall into the Type D class in the general dimensional classification, so that this is a necessary condition. It is also a sufficient condition in four dimensions $[95,96]$ (see also [60]). But it does not appear to be a sufficient condition in higher dimensions 
— the five-dimensional Myers-Perry metrics [122] are Type D [123] and while we showed that the singly-rotating solution has a Weyl doubling formula, the general solution with two rotation parameters does not appear to satisfy such a formula. We also investigated the BPS solution in six-dimensions studied in [124], which is Type D but does not appear to satisfy a doubling formula.

A second way to generate a two-form gauge field strength is if the spacetime admits a closed, covariantly constant four-form which may be used to define a self-dual (or anti-selfdual) two form. These manifolds have special holonomy and the Maxwell field equations then follow from the duality and closure conditions. In the four-dimensional case these are manifolds with SU(2) holonomy. The Gibbons-Hawking metrics provide a broad class of such metrics. We found that in this case the Weyl doubling formula has a correction term which is linear in the gauge field strength - equation (3.10) or equivalently (3.14). This generalisation of doubling could be studied further to see if it applies in other cases. It may also suggest generalisations of the double copy construction. A reader might wonder that such a doubling formula is inevitable given the symmetries of the Weyl tensor. The Gibbons-Hawking case where there is a derivative correction provides a good counter example that demonstrates the non-triviality of the algebraic relation in the algebraic Weyl doubling formula.

In studying a generalisation to higher dimensions an issue arises in that a Weyl tensor constructed from (2.1) using a self-dual two-form is not in general self-dual, unlike in four dimensions. One might have expected that this might be resolved by using self-dual projection operators, but this will not preserve the algebraic symmetries of the tensor in general. Nevertheless we found an example in eight dimensions where a sort of twisted doubling construction exists which expresses the Weyl tensor in terms of a spin(7) self-dual two form. It would be interesting to explore if other examples exist.

A different mechanism for Weyl doubling is if the gauge field strength is an additional field defined on the spacetime, rather than being expressed using the metric and/or Killing vectors. The most natural example of this is the Reissner-Nordström metric in $D$ dimensions. This works in the general case as well as the BPS limit, although perhaps one might have expected that such a construction, requiring the Weyl and Maxwell curvatures to be related, would require the BPS constraint linking the charge and mass. We showed that this discussion generalises to the charged Born-Infeld solution in $D$ dimensions. A recent paper [118] discussed how study of the Born-Infeld electrically charged solution might illuminate the investigation of stringy corrections of the double copy. It would be interesting to see if the doubling approach may provide insights into this.

We then turned to study cases where the gauge field strength is a higher degree $p$-form, and analysed the associated brane solutions. Here it was found that a simple quadratic Weyl doubling formula holds using the $p$-form field strength and contractions of it with the world volume Killing vectors. Evaluating this gives a particularly simple Weyl doubling formula for the components of the Weyl tensor projected on to the world volume. There is a variety of directions for further research, such as how does the inclusion of scalars, Kaluza-Klein reductions and supersymmetry impact the doubling construction. We have studied the Weyl tensor here but there will also be spinor analogues of our formulæ in each dimension (cf. [111]). 
The central question that this paper implicitly poses is, what is the relation to the usual double copy? Can the formulæ for brane solutions be applied to the usual single and double copy? Does the derivative corrected Gibbon-Hawking expression have a conventional double copy interpretation in terms of Maxwell fields in flat space? It was shown in [79] that doubled geometry clarifies the double copy construction for the point charge, relating this to the JNW solution [125]. As we noted in section 6, the Kerr-Schild form in double field theory involves more than one gauge field - for example, two Maxwell fields in the case where there is a Kalb-Ramond field as well as the metric. For the string in five dimensions the $B$ field can indeed be simply constructed from two Maxwell gauge fields. This might also provide insights into exceptional geometry where such a Kerr-Schild formulation is not yet available. One approach following these ideas is to use a DFT or EFT generalised Killing vector as the basis for generating gauge field strengths in the extended space to express the DFT equations.

The phenomenon of Weyl doubling that we have explored here in numerous examples, relating gravity and Abelian gauge theory, reveals a structure that it would be interesting to develop further, and in particular to investigate its relationship to the double copy.

\section{Acknowledgments}

DSB and BS are supported by the U.K. Science and Technology Facilities Council (STFC) with consolidated grant ST/L000415/1, String Theory, Gauge Theory and Duality. RA is supported by a student scholarship from the Ministry of Higher Education of the UAE. We thank Kanghoon Lee, Ricardo Montiero, Malcolm Perry and Chris White for discussions on various aspects of the double copy and classical solutions.

\section{A Myers-Perry pentad}

The pentad used for the analysis of the Myers-Perry metric in subsection 2.2.4 is based on the two null vectors $l^{\mu}=L_{ \pm}^{\mu}$ satisfying the equation $l^{\nu} l^{\rho} C_{\mu \nu \rho[\pi} l_{\omega]}=0[126]$

$$
\begin{aligned}
L_{ \pm}= & \frac{1}{\left(x^{2}-1\right)\left(-1+L^{2} y\right)}\left(\frac{L^{2} x y-y+L^{2} x+1-2 L^{2} y}{x-y} R \partial_{t}-L \partial_{\psi}\right) \\
& \pm \sqrt{\frac{L^{2} x-1}{(x-y)(y-1)}}\left(\partial_{x}+\frac{y^{2}-1}{x^{2}-1} \partial_{y}\right) .
\end{aligned}
$$

We take $l^{\mu}=L_{+}^{\mu}, n^{\mu}=L_{-}^{\mu}$ and choose the three unit norm vectors $m_{i}^{\mu}(i=1,2,3)$ to be

$$
\begin{aligned}
& m_{1}=\sqrt{\frac{(1+x)(x-y)(1+y)\left(-1+L^{2} y\right)^{2}}{\left(1+L^{2}\right) R^{2}(x-1)}}\left(0,-\frac{(x-1)\left(-1+L^{2} x\right)}{(y-1)\left(-1+L^{2} y\right)},-1,0,0\right), \\
& m_{2}=\sqrt{-\frac{(x-y)^{2}}{(1+x)\left(-1+L^{2} x\right)(y-1)^{2} R^{2}}}(0,0,0,-1,0), \\
& m_{3}=\sqrt{-\frac{(x-y)^{2}}{(-1+x)\left(1+L^{2}\right)\left(y^{2}-1\right) R^{2}}}\left(\frac{L R(x-1)(y+1)}{x-y}, 0,0,0,-1\right) .
\end{aligned}
$$


These vectors satisfy the conditions in and beneath (2.41). This choice is convenient in this case as the non-zero weight Weyl-NP (and Maxwell-NP) components vanish directly using them.

Open Access. This article is distributed under the terms of the Creative Commons Attribution License (CC-BY 4.0), which permits any use, distribution and reproduction in any medium, provided the original author(s) and source are credited.

\section{References}

[1] Z. Bern, J.J.M. Carrasco and H. Johansson, New relations for gauge-theory amplitudes, Phys. Rev. D 78 (2008) 085011 [arXiv:0805.3993] [InSPIRE].

[2] Z. Bern, J.J.M. Carrasco and H. Johansson, Perturbative quantum gravity as a double copy of gauge theory, Phys. Rev. Lett. 105 (2010) 061602 [arXiv:1004.0476] [InSPIRE].

[3] Z. Bern, T. Dennen, Y.-T. Huang and M. Kiermaier, Gravity as the square of gauge theory, Phys. Rev. D 82 (2010) 065003 [arXiv:1004.0693] [INSPIRE].

[4] N.E.J. Bjerrum-Bohr, P.H. Damgaard and P. Vanhove, Minimal basis for gauge theory amplitudes, Phys. Rev. Lett. 103 (2009) 161602 [arXiv:0907.1425] [INSPIRE].

[5] S. Stieberger, Open \& closed vs. pure open string disk amplitudes, arXiv:0907.2211 [INSPIRE].

[6] N.E.J. Bjerrum-Bohr, P.H. Damgaard, T. Sondergaard and P. Vanhove, Monodromy and Jacobi-like relations for color-ordered amplitudes, JHEP 06 (2010) 003 [arXiv: 1003.2403] [INSPIRE].

[7] B. Feng, R. Huang and Y. Jia, Gauge amplitude identities by on-shell recursion relation in S-matrix program, Phys. Lett. B 695 (2011) 350 [arXiv: 1004.3417] [INSPIRE].

[8] S.-H. Henry Tye and Y. Zhang, Dual identities inside the gluon and the graviton scattering amplitudes, JHEP 06 (2010) 071 [Erratum ibid. 04 (2011) 114] [arXiv:1003.1732] [INSPIRE].

[9] C.R. Mafra, O. Schlotterer and S. Stieberger, Explicit BCJ numerators from pure spinors, JHEP 07 (2011) 092 [arXiv:1104.5224] [InSPIRE].

[10] R. Monteiro and D. O'Connell, The kinematic algebra from the self-dual sector, JHEP 07 (2011) 007 [arXiv: 1105. 2565] [inSPIRE].

[11] N.E.J. Bjerrum-Bohr, P.H. Damgaard, R. Monteiro and D. O'Connell, Algebras for amplitudes, JHEP 06 (2012) 061 [arXiv:1203.0944] [INSPIRE].

[12] H. Kawai, D.C. Lewellen and S.-H. Henry Tye, A relation between tree amplitudes of closed and open strings, Nucl. Phys. B 269 (1986) 1 [InSPIRE].

[13] Z. Bern, L.J. Dixon, D.C. Dunbar, M. Perelstein and J.S. Rozowsky, On the relationship between Yang-Mills theory and gravity and its implication for ultraviolet divergences, Nucl. Phys. B 530 (1998) 401 [hep-th/9802162] [INSPIRE].

[14] M.B. Green, J.H. Schwarz and L. Brink, $N=4$ Yang-Mills and $N=8$ supergravity as limits of string theories, Nucl. Phys. B 198 (1982) 474 [INSPIRE].

[15] Z. Bern, J.S. Rozowsky and B. Yan, Two loop four gluon amplitudes in $N=4$ super Yang-Mills, Phys. Lett. B 401 (1997) 273 [hep-ph/9702424] [INSPIRE]. 
[16] J.J.M. Carrasco and H. Johansson, Five-point amplitudes in $N=4$ super-Yang-Mills theory and $N=8$ supergravity, Phys. Rev. D 85 (2012) 025006 [arXiv:1106.4711] [InSPIRE].

[17] J.J.M. Carrasco, M. Chiodaroli, M. Günaydin and R. Roiban, One-loop four-point amplitudes in pure and matter-coupled $N=4$ supergravity, JHEP 03 (2013) 056 [arXiv:1212.1146] [INSPIRE].

[18] C.R. Mafra and O. Schlotterer, The structure of n-point one-loop open superstring amplitudes, JHEP 08 (2014) 099 [arXiv: 1203.6215] [INSPIRE].

[19] R.H. Boels, R.S. Isermann, R. Monteiro and D. O'Connell, Colour-kinematics duality for one-loop rational amplitudes, JHEP 04 (2013) 107 [arXiv: 1301.4165] [INSPIRE].

[20] N.E.J. Bjerrum-Bohr, T. Dennen, R. Monteiro and D. O'Connell, Integrand oxidation and one-loop colour-dual numerators in $N=4$ gauge theory, JHEP 07 (2013) 092 [arXiv: 1303.2913] [INSPIRE].

[21] Z. Bern, S. Davies, T. Dennen, Y.-T. Huang and J. Nohle, Color-kinematics duality for pure Yang-Mills and gravity at one and two loops, Phys. Rev. D 92 (2015) 045041 [arXiv:1303.6605] [INSPIRE].

[22] Z. Bern, S. Davies and T. Dennen, The ultraviolet structure of half-maximal supergravity with matter multiplets at two and three loops, Phys. Rev. D 88 (2013) 065007 [arXiv: 1305.4876] [INSPIRE].

[23] J. Nohle, Color-kinematics duality in one-loop four-gluon amplitudes with matter, Phys. Rev. D 90 (2014) 025020 [arXiv: 1309.7416] [inSPIRE].

[24] Z. Bern, S. Davies, T. Dennen, A.V. Smirnov and V.A. Smirnov, Ultraviolet properties of $N=4$ supergravity at four loops, Phys. Rev. Lett. 111 (2013) 231302 [arXiv:1309.2498] [INSPIRE].

[25] S.G. Naculich, H. Nastase and H.J. Schnitzer, All-loop infrared-divergent behavior of most-subleading-color gauge-theory amplitudes, JHEP 04 (2013) 114 [arXiv:1301.2234] [INSPIRE].

[26] Y.-J. Du, B. Feng and C.-H. Fu, Dual-color decompositions at one-loop level in Yang-Mills theory, JHEP 06 (2014) 157 [arXiv: 1402.6805] [INSPIRE].

[27] C.R. Mafra and O. Schlotterer, Towards one-loop SYM amplitudes from the pure spinor BRST cohomology, Fortsch. Phys. 63 (2015) 105 [arXiv:1410.0668] [INSPIRE].

[28] Z. Bern, S. Davies and T. Dennen, Enhanced ultraviolet cancellations in $N=5$ supergravity at four loops, Phys. Rev. D 90 (2014) 105011 [arXiv:1409.3089] [INSPIRE].

[29] C.R. Mafra and O. Schlotterer, Two-loop five-point amplitudes of super Yang-Mills and supergravity in pure spinor superspace, JHEP 10 (2015) 124 [arXiv:1505.02746] [INSPIRE].

[30] S. He, R. Monteiro and O. Schlotterer, String-inspired BCJ numerators for one-loop MHV amplitudes, JHEP 01 (2016) 171 [arXiv:1507.06288] [INSPIRE].

[31] Z. Bern, S. Davies and J. Nohle, Double-copy constructions and unitarity cuts, Phys. Rev. D 93 (2016) 105015 [arXiv:1510.03448] [INSPIRE].

[32] G. Mogull and D. O'Connell, Overcoming obstacles to colour-kinematics duality at two loops, JHEP 12 (2015) 135 [arXiv:1511.06652] [INSPIRE].

[33] M. Chiodaroli, M. Günaydin, H. Johansson and R. Roiban, Spontaneously broken Yang-Mills-Einstein supergravities as double copies, JHEP 06 (2017) 064 [arXiv: 1511.01740] [INSPIRE]. 
[34] Z. Bern, J.J.M. Carrasco, W.-M. Chen, H. Johansson, R. Roiban and M. Zeng, Five-loop four-point integrand of $N=8$ supergravity as a generalized double copy, Phys. Rev. D 96 (2017) 126012 [arXiv: 1708.06807] [INSPIRE].

[35] H. Johansson and A. Ochirov, Color-kinematics duality for QCD amplitudes, JHEP 01 (2016) 170 [arXiv: 1507.00332] [INSPIRE].

[36] S. Oxburgh and C.D. White, BCJ duality and the double copy in the soft limit, JHEP 02 (2013) 127 [arXiv: 1210.1110] [INSPIRE].

[37] C.D. White, Factorization properties of soft graviton amplitudes, JHEP 05 (2011) 060 [arXiv: 1103.2981] [INSPIRE].

[38] S. Melville, S.G. Naculich, H.J. Schnitzer and C.D. White, Wilson line approach to gravity in the high energy limit, Phys. Rev. D 89 (2014) 025009 [arXiv:1306.6019] [INSPIRE].

[39] A. Luna, S. Melville, S.G. Naculich and C.D. White, Next-to-soft corrections to high energy scattering in QCD and gravity, JHEP 01 (2017) 052 [arXiv: 1611.02172] [INSPIRE].

[40] R. Saotome and R. Akhoury, Relationship between gravity and gauge scattering in the high energy limit, JHEP 01 (2013) 123 [arXiv:1210.8111] [INSPIRE].

[41] A. Sabio Vera, E. Serna Campillo and M.A. Vazquez-Mozo, Color-kinematics duality and the Regge limit of inelastic amplitudes, JHEP 04 (2013) 086 [arXiv:1212.5103] [INSPIRE].

[42] H. Johansson, A. Sabio Vera, E. Serna Campillo and M.A. Vázquez-Mozo, Color-kinematics duality in multi-Regge kinematics and dimensional reduction, JHEP 10 (2013) 215 [arXiv: 1307.3106] [INSPIRE].

[43] H. Johansson, A. Sabio Vera, E. Serna Campillo and M.A. Vazquez-Mozo, Color-kinematics duality and dimensional reduction for graviton emission in Regge limit, in International workshop on low X physics, (2013) [arXiv:1310.1680] [INSPIRE].

[44] R. Monteiro, D. O'Connell and C.D. White, Black holes and the double copy, JHEP 12 (2014) 056 [arXiv: 1410.0239] [INSPIRE].

[45] A. Luna, R. Monteiro, D. O'Connell and C.D. White, The classical double copy for Taub-NUT spacetime, Phys. Lett. B 750 (2015) 272 [arXiv:1507.01869] [INSPIRE].

[46] D.S. Berman, E. Chacón, A. Luna and C.D. White, The self-dual classical double copy, and the Eguchi-Hanson instanton, JHEP 01 (2019) 107 [arXiv: 1809.04063] [INSPIRE].

[47] S. Sabharwal and J.W. Dalhuisen, Anti-self-dual spacetimes, gravitational instantons and knotted zeros of the Weyl tensor, JHEP 07 (2019) 004 [arXiv: 1904.06030] [INSPIRE].

[48] A. Anastasiou, L. Borsten, M.J. Duff, L.J. Hughes and S. Nagy, Yang-Mills origin of gravitational symmetries, Phys. Rev. Lett. 113 (2014) 231606 [arXiv:1408.4434] [INSPIRE].

[49] L. Borsten and M.J. Duff, Gravity as the square of Yang-Mills?, Phys. Scripta 90 (2015) 108012 [arXiv: 1602.08267] [INSPIRE].

[50] A. Anastasiou et al., Twin supergravities from Yang-Mills theory squared, Phys. Rev. D 96 (2017) 026013 [arXiv:1610.07192] [INSPIRE].

[51] A. Anastasiou, L. Borsten, M.J. Duff, A. Marrani, S. Nagy and M. Zoccali, Are all supergravity theories Yang-Mills squared?, Nucl. Phys. B 934 (2018) 606 [arXiv: 1707.03234] [INSPIRE].

[52] G.L. Cardoso, S. Nagy and S. Nampuri, A double copy for $N=2$ supergravity: a linearised tale told on-shell, JHEP 10 (2016) 127 [arXiv:1609.05022] [INSPIRE]. 
[53] L. Borsten, $D=6, N=(2,0)$ and $N=(4,0)$ theories, Phys. Rev. D 97 (2018) 066014 [arXiv: 1708.02573] [INSPIRE].

[54] A. Anastasiou, L. Borsten, M.J. Duff, A. Marrani, S. Nagy and M. Zoccali, The mile high magic pyramid, Contemp. Math. 721 (2019) 1 [arXiv:1711.08476] [INSPIRE].

[55] A. Anastasiou, L. Borsten, M.J. Duff, S. Nagy and M. Zoccali, Gravity as gauge theory squared: a ghost story, Phys. Rev. Lett. 121 (2018) 211601 [arXiv: 1807.02486] [InSPIRE].

[56] G. Lopes Cardoso, G. Inverso, S. Nagy and S. Nampuri, Comments on the double copy construction for gravitational theories, PoS (CORFU2017) 177 (2018) [arXiv:1803.07670] [INSPIRE].

[57] M. Carrillo González, R. Penco and M. Trodden, Radiation of scalar modes and the classical double copy, JHEP 11 (2018) 065 [arXiv: 1809.04611] [INSPIRE].

[58] M. Gurses and B. Tekin, Classical double copy: Kerr-Schild-Kundt metrics from Yang-Mills theory, Phys. Rev. D 98 (2018) 126017 [arXiv:1810.03411] [InSPIRE].

[59] F. Brown and C. Dupont, Single-valued integration and double copy, arXiv:1810.07682 [INSPIRE].

[60] A. Luna, R. Monteiro, I. Nicholson and D. O'Connell, Type D spacetimes and the Weyl double copy, Class. Quant. Grav. 36 (2019) 065003 [arXiv:1810.08183] [INSPIRE].

[61] J. Faller and J. Plefka, Positive helicity Einstein-Yang-Mills amplitudes from the double copy method, Phys. Rev. D 99 (2019) 046008 [arXiv: 1812.04053] [INSPIRE].

[62] J.A. Farrow, A.E. Lipstein and P. McFadden, Double copy structure of CFT correlators, JHEP 02 (2019) 130 [arXiv:1812.11129] [INSPIRE].

[63] C.R. Mafra and O. Schlotterer, Towards the n-point one-loop superstring amplitude. Part III. One-loop correlators and their double-copy structure, JHEP 08 (2019) 092 [arXiv: 1812.10971] [INSPIRE].

[64] Y.F. Bautista and A. Guevara, From scattering amplitudes to classical physics: universality, double copy and soft theorems, arXiv:1903.12419 [INSPIRE].

[65] M. Carrillo González, B. Melcher, K. Ratliff, S. Watson and C.D. White, The classical double copy in three spacetime dimensions, JHEP 07 (2019) 167 [arXiv:1904.11001] [INSPIRE].

[66] W. Cho and K. Lee, Heterotic Kerr-Schild double field theory and classical double copy, JHEP 07 (2019) 030 [arXiv: 1904.11650] [INSPIRE].

[67] J. Plefka, C. Shi, J. Steinhoff and T. Wang, Breakdown of the classical double copy for the effective action of dilaton-gravity at NNLO, Phys. Rev. D 100 (2019) 086006 [arXiv: 1906.05875] [INSPIRE].

[68] H. Johansson and A. Ochirov, Double copy for massive quantum particles with spin, JHEP 09 (2019) 040 [arXiv: 1906.12292] [INSPIRE].

[69] A.P.V. and A. Manu, Classical double copy from color kinematics duality: a proof in the soft limit, Phys. Rev. D 101 (2020) 046014 [arXiv:1907.10021] [INSPIRE].

[70] Z. Bern, C. Cheung, R. Roiban, C.-H. Shen, M.P. Solon and M. Zeng, Black hole binary dynamics from the double copy and effective theory, JHEP 10 (2019) 206 [arXiv: 1908.01493] [INSPIRE]. 
[71] M. Carrillo González, R. Penco and M. Trodden, Shift symmetries, soft limits, and the double copy beyond leading order, arXiv:1908.07531 [INSPIRE].

[72] Y.F. Bautista and A. Guevara, On the double copy for spinning matter, arXiv:1908.11349 [INSPIRE].

[73] A.R. Fazio, Cosmological correlators, in-in formalism and double copy, Mod. Phys. Lett. A 35 (2020) 2050076 [arXiv: 1909. 07343] [INSPIRE].

[74] I. Bah, R. Dempsey and P. Weck, Kerr-Schild double copy and complex worldlines, JHEP 02 (2020) 180 [arXiv: 1910.04197] [INSPIRE].

[75] Y.-T. Huang, U. Kol and D. O'Connell, Double copy of electric-magnetic duality, Phys. Rev. D 102 (2020) 046005 [arXiv: 1911.06318] [INSPIRE].

[76] J. Plefka, C. Shi and T. Wang, Double copy of massive scalar QCD, Phys. Rev. D 101 (2020) 066004 [arXiv: 1911.06785] [inSPIRE].

[77] R. Alawadhi, D.S. Berman, B. Spence and D. Peinador Veiga, S-duality and the double copy, JHEP 03 (2020) 059 [arXiv: 1911.06797] [INSPIRE].

[78] W.D. Goldberger and J. Li, Strings, extended objects, and the classical double copy, JHEP 02 (2020) 092 [arXiv:1912.01650] [INSPIRE].

[79] K. Kim, K. Lee, R. Monteiro, I. Nicholson and D. Peinador Veiga, The classical double copy of a point charge, JHEP 02 (2020) 046 [arXiv: 1912.02177] [INSPIRE].

[80] A.E. Lipstein and P. McFadden, Double copy structure and the flat space limit of conformal correlators in even dimensions, Phys. Rev. D 101 (2020) 125006 [arXiv:1912.10046] [INSPIRE].

[81] L. Borsten, I. Jubb, V. Makwana and S. Nagy, Gauge $\times$ gauge on spheres, JHEP 06 (2020) 096 [arXiv: 1911.12324] [INSPIRE].

[82] N. Bahjat-Abbas, R. Stark-Muchão and C.D. White, Monopoles, shockwaves and the classical double copy, JHEP 04 (2020) 102 [arXiv: 2001.09918] [INSPIRE].

[83] C. Cheung and G.N. Remmen, Entanglement and the double copy, JHEP 05 (2020) 100 [arXiv: 2002.10470] [INSPIRE].

[84] L. Alfonsi, C.D. White and S. Wikeley, Topology and Wilson lines: global aspects of the double copy, JHEP 07 (2020) 091 [arXiv: 2004.07181] [INSPIRE].

[85] A. Momeni, J. Rumbutis and A.J. Tolley, Massive gravity from double copy, arXiv: 2004.07853 [INSPIRE].

[86] A. Luna, S. Nagy and C. White, The convolutional double copy: a case study with a point, arXiv:2004.11254 [INSPIRE].

[87] L.A. Johnson, C.R.T. Jones and S. Paranjape, Constraints on a massive double-copy and applications to massive gravity, arXiv:2004.12948 [INSPIRE].

[88] L. Borsten and S. Nagy, The pure BRST Einstein-Hilbert Lagrangian from the double-copy to cubic order, JHEP 07 (2020) 093 [arXiv: 2004.14945] [INSPIRE].

[89] E. Lescano and J.A. Rodríguez, $N=1$ supersymmetric double field theory and the generalized Kerr-Schild ansatz, arXiv:2002.07751 [INSPIRE].

[90] N. Moynihan and J. Murugan, On-shell electric-magnetic duality and the dual graviton, arXiv:2002.11085 [INSPIRE]. 
[91] C. Keeler, T. Manton and N. Monga, From Navier-Stokes to Maxwell via Einstein, JHEP 08 (2020) 147 [arXiv : 2005. 04242] [inSPIRE].

[92] N. Moynihan, Scattering amplitudes and the double copy in topologically massive theories, arXiv:2006 .15957 [INSPIRE].

[93] T. Adamo and A. Ilderton, Classical and quantum double copy of back-reaction, arXiv:2005.05807 [INSPIRE].

[94] M. Mars, Space-time Ehlers group: transformation law for the Weyl tensor, Class. Quant. Grav. 18 (2001) 719 [gr-qc/0101020] [INSPIRE].

[95] M. Walker and R. Penrose, On quadratic first integrals of the geodesic equations for type [22] spacetimes, Commun. Math. Phys. 18 (1970) 265 [INSPIRE].

[96] L.P. Hughston, R. Penrose, P. Sommers and M. Walker, On a quadratic first integral for the charged particle orbits in the charged Kerr solution, Commun. Math. Phys. 27 (1972) 303 [INSPIRE].

[97] V. Frolov, P. Krtous and D. Kubiznak, Black holes, hidden symmetries, and complete integrability, Living Rev. Rel. 20 (2017) 6 [arXiv:1705.05482] [INSPIRE].

[98] L.J. Mason and A. Taghavi-Chabert, Killing-Yano tensors and multi-Hermitian structures, J. Geom. Phys. 60 (2010) 907 [arXiv:0805.3756] [InSPIRE].

[99] M. Ortaggio, V. Pravda and A. Pravdova, Algebraic classification of higher dimensional spacetimes based on null alignment, Class. Quant. Grav. 30 (2013) 013001 [arXiv:1211.7289] [INSPIRE].

[100] H.S. Reall, Algebraically special solutions in higher dimensions, in Black holes in higher dimensions, G.T. Horowitz ed., (2012), pg. 213 [arXiv:1105.4057] [INSPIRE].

[101] A.E.K. Lim and J. Carminati, The determination of all Syzygies for the dependent polynomial invariants of the Riemann tensor. I. Pure Ricci and pure Weyl invariants, J. Math. Phys. 45 (2004) 1673.

[102] T. Ortín, Gravity and strings, second edition, Cambridge University Press, Cambridge, U.K. (2015) [INSPIRE].

[103] J.F. Plebanski and M. Demianski, Rotating, charged, and uniformly accelerating mass in general relativity, Annals Phys. 98 (1976) 98 [INSPIRE].

[104] J.B. Griffiths and J. Podolsky, A new look at the Plebanski-Demianski family of solutions, Int. J. Mod. Phys. D 15 (2006) 335 [gr-qc/0511091] [INSPIRE].

[105] H. Elvang and R. Emparan, Black rings, supertubes, and a stringy resolution of black hole nonuniqueness, JHEP 11 (2003) 035 [hep-th/0310008] [INSPIRE].

[106] R. Emparan and H.S. Reall, A rotating black ring solution in five-dimensions, Phys. Rev. Lett. 88 (2002) 101101 [hep-th/0110260] [INSPIRE].

[107] R. Milson, A. Coley, V. Pravda and A. Pravdova, Alignment and algebraically special tensors in Lorentzian geometry, Int. J. Geom. Meth. Mod. Phys. 2 (2005) 41 [gr-qc/0401010] [INSPIRE].

[108] A. Coley, R. Milson, V. Pravda and A. Pravdova, Classification of the Weyl tensor in higher dimensions, Class. Quant. Grav. 21 (2004) L35 [gr-qc/0401008] [INSPIRE].

[109] P.-J. De Smet, Black holes on cylinders are not algebraically special, Class. Quant. Grav. 19 (2002) 4877 [hep-th/0206106] [INSPIRE]. 
[110] M. Godazgar, Spinor classification of the Weyl tensor in five dimensions, Class. Quant. Grav. 27 (2010) 245013 [arXiv: 1008.2955] [INSPIRE].

[111] R. Monteiro, I. Nicholson and D. O'Connell, Spinor-helicity and the algebraic classification of higher-dimensional spacetimes, Class. Quant. Grav. 36 (2019) 065006 [arXiv: 1809.03906] [INSPIRE].

[112] A.M. Ghezelbash and R. Oraji, Gibbons-Hawking M-branes, JHEP 12 (2009) 039 [arXiv:0908.3160] [INSPIRE].

[113] E. Corrigan, C. Devchand, D.B. Fairlie and J. Nuyts, First order equations for gauge fields in spaces of dimension greater than four, Nucl. Phys. B 214 (1983) 452 [InSPIRE].

[114] S.S. Gubser, TASI lectures: special holonomy in string theory and M-theory, in Theoretical Advanced Study Institute in Elementary Particle Physics (TASI 2001): strings, branes and EXTRA dimensions, (2002), pg. 197 [hep-th/0201114] [INSPIRE].

[115] B.S. Acharya, M. O'Loughlin and B.J. Spence, Higher dimensional analogs of Donaldson-Witten theory, Nucl. Phys. B 503 (1997) 657 [hep-th/9705138] [INSPIRE].

[116] S. Salur and O. Santillan, New Spin(7) holonomy metrics admiting $G_{2}$ holonomy reductions and M-theory/IIA dualities, Phys. Rev. D 79 (2009) 086009 [arXiv:0811.4422] [INSPIRE].

[117] D.A. Rasheed, Nonlinear electrodynamics: zeroth and first laws of black hole mechanics, hep-th/9702087 [INSPIRE].

[118] O. Pasarin and A.A. Tseytlin, Generalised Schwarzschild metric from double copy of point-like charge solution in Born-Infeld theory, Phys. Lett. B 807 (2020) 135594 [arXiv: 2005.12396] [INSPIRE].

[119] K.S. Stelle, BPS branes in supergravity, in ICTP summer school in high-energy physics and cosmology, (1998) [hep-th/9803116] [INSPIRE].

[120] K. Lee, Kerr-Schild double field theory and classical double copy, JHEP 10 (2018) 027 [arXiv: 1807.08443] [INSPIRE].

[121] D.S. Berman and C.D.A. Blair, The geometry, branes and applications of exceptional field theory, arXiv:2006.09777 [INSPIRE].

[122] R.C. Myers, Myers-Perry black holes, in Black holes in higher dimensions, G.T. Horowitz ed., (2012), pg. 101 [arXiv:1111.1903] [InSPIRE].

[123] P.-J. De Smet, The Petrov type of the five-dimensional Myers-Perry metric, Gen. Rel. Grav. 36 (2004) 1501 [gr-qc/0312021] [INSPIRE].

[124] W. Chen, H. Lü and C.N. Pope, General Kerr-NUT-AdS metrics in all dimensions, Class. Quant. Grav. 23 (2006) 5323 [hep-th/0604125] [INSPIRE].

[125] A.I. Janis, E.T. Newman and J. Winicour, Reality of the Schwarzschild singularity, Phys. Rev. Lett. 20 (1968) 878 [INSPIRE].

[126] V. Pravda and A. Pravdova, WANDs of the black ring, Gen. Rel. Grav. 37 (2005) 1277 [gr-qc/0501003] [INSPIRE]. 